\title{
Ecological Models of Language Competition
}

\author{
Anne Kandler \\ James Steele \\ AHRC Centre for the Evolution of Cultural Diversity \\ Institute of Archaeology \\ University College London \\ London, UK \\ a.kandler@ucl.ac.uk \\ j.steele@ucl.ac.uk
}

\begin{abstract}
The contemporary global language "extinction crisis" has been analyzed by several influential linguists using concepts from ecology. In this article we study different reaction-diffusion models to explain the dynamics of language competition. We are mainly interested in situations where one language has a status advantage compared with the other. We consider previous applications of competition models from ecology, with particular attention to the implications of the "carrying capacity" term in such models. We derive existence as well as stability conditions for the equilibria of the models considered, and show that depending on environmental conditions both situations - coexistence of competing languages or extinction of the less advantageous language-are possible.
\end{abstract}

\section{Keywords}

carrying capacity, language coexistence, language competition, language extinction, mathematical modeling, reactiondiffusion competition model, spatial heterogeneity 
It is estimated that perhaps half of the world's 6000-7000 languages will be lost during the next 100 years (Krauss 1992). The rapid evolution of today's "language landscape" is influenced by a number of different economic, political, and cultural factors. In the course of globalization and of recent trends for urbanization and long-distance economic migration, interactions between groups speaking different languages have increased and so has the need for a common language of communication (Baggs and Freedman 1993). Some languages (e.g., English) have come to fill that role for historical, economic, and hegemonic reasons, and as a consequence have risen in importance in official and nonofficial matters. Their lexicons have consequently expanded to represent all the paraphernalia of modernization, further enhancing their competitive advantage. In contrast, minority languages are particularly subject to pressure and at risk of extinction, mainly because speakers perceive an economic gain from shifting (Mufwene 2002).

A number of prominent linguists have called for an ecological approach to this global linguistic "extinction crisis." However, they differ on what is meant by an ecological approach. Eco-linguistics (as represented by Muhlhausler 2000) understands an ecological system to be one in which there is self-organized structural diversity based on primarily beneficial interactions among entities (e.g., languages) occupying different niches. Language planning in this context is taken to mean maintenance by "habitat preservation" of diversity among languages that retain equal status. This is motivated by the Whorfian belief that each language encodes a unique (and locally adapted) way of perceiving the environment. Mufwene (2002), in contrast, understands an ecological approach to the language landscape to be primarily characterized by selection and competition dynamics. Language birth and death are seen as fundamentally dynamic processes, driven in part by the adaptive decisions of speakers to maintain or shift languages to match their own evolving socioeconomic circumstances.

In this article we address the problem of characterizing and modeling the dynamics of language competition in the light of such linguistic perspectives. Abrams and Strogatz (2003) presented a simple two-language competition model to explain historical data on the decline of endangered languages, and subsequently a number of very interesting extensions of their model have been published. The Abrams and Strogatz model (2003) proposes that the attractiveness of a language increases with the number of speakers and with its perceived status, defined as a reflection of the social and economic opportunities afforded to its speakers. In this model, the extinction of the language with the lower social status is inevitable. A generalization of this approach was made by Patriarca and Leppänen (2004). They introduced spatial dependence, which results in a reaction-diffusion equation. They found a possibility for preservation of both languages under the assumption that they are initially separated in space. In the equilibrium state of their model, the languages are concentrated in different zones and interact only in a narrow transition region (Patriarca and Leppänen 2004). In this model, too, languageshifting rates reflect the perceived status of each language. In both these models, status-dependent and density-dependent shifting rates govern the competition dynamic, and the system is closed with respect to overall population size (i.e., the total number of speakers is conserved).

Subsequently, to explain cases where both languages survive in only one zone of competition, Pinasco and Romanelli (2006) developed an ecological model of Lotka-Volterra type. This model introduced an additional growth rate term, whereby new speakers of each language are recruited not just by shifting, but also by reproduction. In order to maintain finite population sizes, reproduction is modeled as a logistic process in which net growth falls off as the population of speakers of each language approaches its limit (or "carrying capacity"). Pinasco and Romanelli (2006) found that a steady state was possible in which both languages coexist without spatial separation, when the reproductive rate of speakers of the subordinate language balances the loss rate due to language-shifting. ${ }^{1}$

The aim of this article is to extend and modify the ecological model of language competition with a special focus on the interpretation and usage of the term "carrying capacity." Following Abrams and Strogatz (2003) we make the simplifying assumption that languages are fixed in form, and competing with each other for speakers. Such simplifying assumptions help us to understand the fundamental competition dynamics: as a general rule it is better to start with a simple model with a few key variables, and only add extra dimensions if they significantly increase explanatory power. We describe the interaction and growth dynamics of populations of speakers of two competing languages in different reaction-diffusion competition models and are mainly interested in situations where one language has higher status (and is more likely to be the target of language-shifters). We will see that the ecological competition model has some deficiencies when applied to the case of language competition and demonstrate how this model can be modified to obtain better results. We are interested both in the determination of language equilibria, which express the stable long-term ratio of the competing languages, and in the time taken for the equilibria to be reached.

In this article we assume the simplest possible shift mechanism. Speakers are able to shift languages immediately without passing through a state where both languages are spoken at the same time. We are aware of the need of incorporating bilingualism to produce more realistic models but here we want to focus on the extension and modification of existing mathematical models of language shift. In a forthcoming publication the authors will analyze a more complex model which considers the effects of bilingualism. 


\section{Standard Ecological Model}

Following Pinasco and Romanelli (2006), we initially model the dynamics of two competing languages by the reactiondiffusion competition model

$$
\begin{aligned}
& \frac{\partial u_{1}}{\partial t}=d_{1} \Delta u_{1}+u_{1}\left[a_{1}-b_{1} u_{1}+c u_{2}\right], \\
& \frac{\partial u_{2}}{\partial t}=d_{2} \Delta u_{2}+u_{2}\left[a_{2}-b_{2} u_{2}-c u_{1}\right]
\end{aligned}
$$

with the boundary conditions $\partial u_{1} / \partial n=0, \partial u_{2} / \partial n=0$, for $u_{1}, u_{2} \in \partial D$. These boundary conditions describe the situation of a finite environment (a bounded convex two-dimensional domain $D$ ), with no diffusion possible beyond its boundary $\partial D$. The time- and space-dependent variables $u_{1}$ and $u_{2}$ stand for the frequencies of speakers of languages 1 and 2, respectively. The terms $\partial u_{1} / \partial t$ and $\partial u_{2} / \partial t$ indicate the temporal change of the frequencies and are determined respectively by $d_{1} \Delta u_{1}+u_{1}\left[a_{1}-b_{1} u_{1}+c u_{2}\right]$ and $d_{2} \Delta u_{2}+u_{2}\left[a_{2}-b_{2} u_{2}-\right.$ $\left.c u_{1}\right]$. The diffusion components $d_{1} \Delta u_{1}$ and $d_{2} \Delta u_{2}$ model the spread of speakers in space based on a random walk with density-dependent mixing ( $\Delta$ is the Laplace operator). The terms $u_{1}\left[a_{1}-b_{1} u_{1}+c u_{2}\right]$ and $u_{2}\left[a_{2}-b_{2} u_{2}-c u_{1}\right]$ describe the growth in number of the speakers of each language. In this case the growth of the population of speakers of one language is not independent of the other. The influence is modeled by the conversion term $c u_{1} u_{2}$ with a conversion rate $c$. This means that at each time step, a proportion $c u_{1} u_{2}$ of speakers of language 2 convert to language 1 . In terms of Abrams and Strogatz (2003) the coefficient $c$ can be interpreted as the status advantage of language 1 and reflects the social and economic opportunities afforded to its speakers. Since we consider only two languages, an increase in the number of speakers of language 1 results in an equal decrease in the number of speakers of language 2 . In the absence of competition, i.e., when $c u_{1} u_{2}=0$, the growth dynamics are reduced to logistic growth. The coefficients $a_{1}$ and $a_{2}$ express the intrinsic growth rate of the number of speakers of each language, and $b_{1}$ and $b_{2}$ the self-limiting or crowding coefficients. In ecology, where such models represent competition for resources between two species, the self-limiting coefficient is described as an environmental "carrying capacity" $K_{i}, i=1,2$, or an upper boundary for population size (Ehrlich 1994), where the following relations yield

$$
u_{i}\left[a_{i}-b_{i} u_{i}\right]=a_{i} u_{i}\left[1-\frac{u_{i}}{K_{i}}\right] \text { with } K_{i}=a_{i} / b_{i}, i=1,2 .
$$

Figure 1 illustrates the logistic growth pattern of a single population. For this example we have chosen a growth coefficient $a=1$ and a carrying capacity $K=1$. The dashed line is the exponential or Malthusian growth, which is characterized as

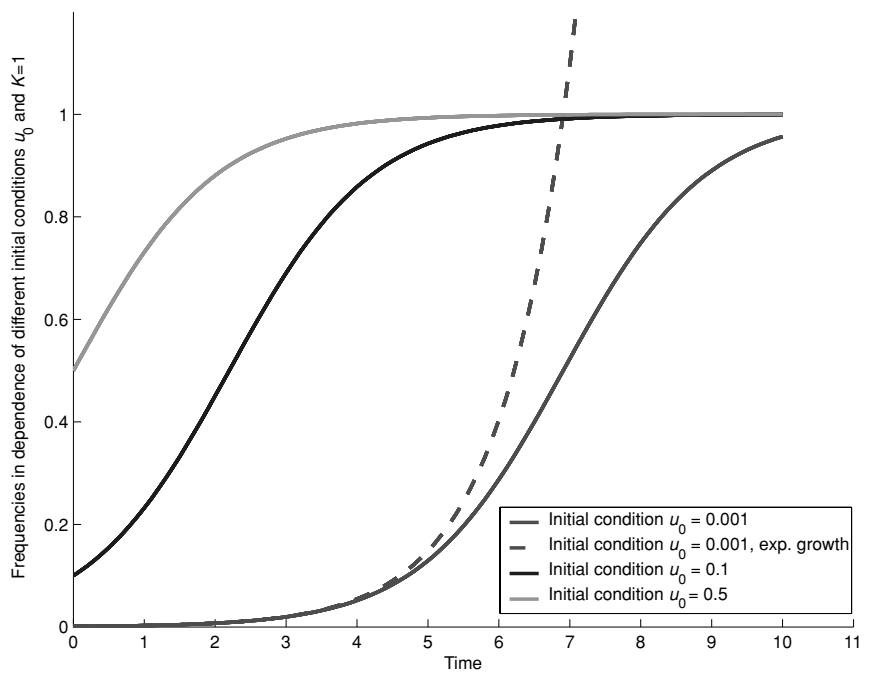

Figure 1.

Logistic growth with initial frequencies $u_{0}=0.001,0.1,0.5$.

unrestricted growth. In that case the self-limiting coefficient $b=a / K$ is equal to zero. The solid lines show the logistic growth assuming different initial values. It is obvious that all lines converge toward the carrying capacity. The self-limiting coefficient $b=a / K$ is responsible for that behavior.

At this point, we may ask whether it is reasonable that both languages should possess separate carrying capacities for their speakers, $K_{1}$ and $K_{2}$, in model (1). In the original ecological context, where two competing species cannot interbreed, the key question is how they partition the available resources. In such situations, the optimal outcome for any single species would be to reach its carrying capacity, without interference from competitors. In the case of language competition, however, speakers can shift languages, and the system is one in which alternative learned behavioral traits are subject to selection in a single population of speakers. We will turn back to this critical point in a later section and present a modified version of model (1), which is based on the assumption that there is only one carrying capacity $K$ describing the maximum population size regardless of the language spoken.

We now analyze the implications of model (1) in more detail. To determine the long-term ratios of languages 1 and 2 we have to look for equilibria to which the language frequencies are attracted. It is well known (e.g., Cosner and Lazer 1984; Pinasco and Romanelli 2006) that for model (1) in a bounded two-dimensional habitat $D$ the following four equilibria ${ }^{2}$ are obtained:

$$
\begin{aligned}
& \left(u_{1}^{*}, u_{2}^{*}\right)=(0,0), \quad\left(u_{1}^{*}, u_{2}^{*}\right)=\left(K_{1}, 0\right), \\
& \left(u_{1}^{*}, u_{2}^{*}\right)=\left(0, K_{2}\right), \\
& \left(u_{1}^{*}, u_{2}^{*}\right)=\left(\frac{a_{2} K_{1}\left(a_{1}+c K_{2}\right)}{a_{1} a_{2}+c^{2} K_{1} K_{2}}, \frac{a_{1} K_{2}\left(a_{2}-c K_{1}\right)}{a_{1} a_{2}+c^{2} K_{1} K_{2}}\right) .
\end{aligned}
$$


The first equilibrium means simply that no speakers of either language survive, and this is of no interest here. The second and third states describe situations where one language has become extinct. If the fourth equilibrium is positive in both components, then it is referred as a coexistence state. Generally, coexistence states are very interesting situations since-as the name suggests - both languages can exist together. But in the case considered here the coexistence case is not feasible since it exists only when either $u_{1}^{*}>K_{1}$ or $u_{2}^{*}<0$. This equilibrium requires that if the frequency of language 1 is less than the carrying capacity $K_{1}$, then the frequency of language 2 is negative, which is not realistic. And in contrast, if the frequency of language 2 is positive then the frequency of language 1 must be greater than the carrying capacity $K_{1}$, which is also not consistent with the definition of this parameter (since, in effect, $K_{1}=u_{1}^{\max }$ ). So we obtain no steady state of coexistence in model (1).

After identifying possible equilibria we have to identify which (if any) states are stable. In this context the model parameters $a_{1}, a_{2}, b_{1}, b_{2}, c$ play a crucial role-different environmental conditions will support different equilibria. The proof of stability can be done by phase-plane analysis (e.g., Murray 2002) and the following results are obtained. The equilibria $(0,0)$ and $\left(0, K_{2}\right)$ are never stable. Further $\left(K_{1}, 0\right)$ is stable for the parameter constellation $a_{2}<c K_{1}$. But unfortunately if we look at the time course to this state we observe that it is not feasible either, since the frequency of language 1 will exceed the carrying capacity $K_{1}$ at some time and this violation of the restriction on maximum numbers is not only a short-term effect while transient overcrowding is regulated. This problem is independent of initial conditions or parameter values. It occurs due to the incorporation into a two-species competition model of a new switching term. To illustrate this fact we consider an example with the parameter constellation

$$
\begin{aligned}
& d_{1}=0.005, \quad d_{2}=0.001, \quad a_{1}=0.001, \quad a_{2}=0.01 \\
& K_{1}=K_{2}=1, \quad c=0.15
\end{aligned}
$$

and for simplicity the habitat $D$ is chosen as a rectangle $D=[0,1] \times[0,1]$. The parameters $a_{1}, a_{2}$ describe the relative population growth per year and the conversion rate $c=0.15$ is assumed to be moderate. Figures $2-5$ show the frequencies of both languages as a cut through the rectangular domain at different times. The initial conditions are as in Figure 2, the languages are spatially separated, and speakers of the dominant language 1 are initially less abundant.

With ongoing time we observe the impacts of diffusion, growth, and interaction and as a result the dominance of language 1. Language 2 becomes extinct after a finite time period (Figure 5). But Figure 4 shows that language 1 exceeds its

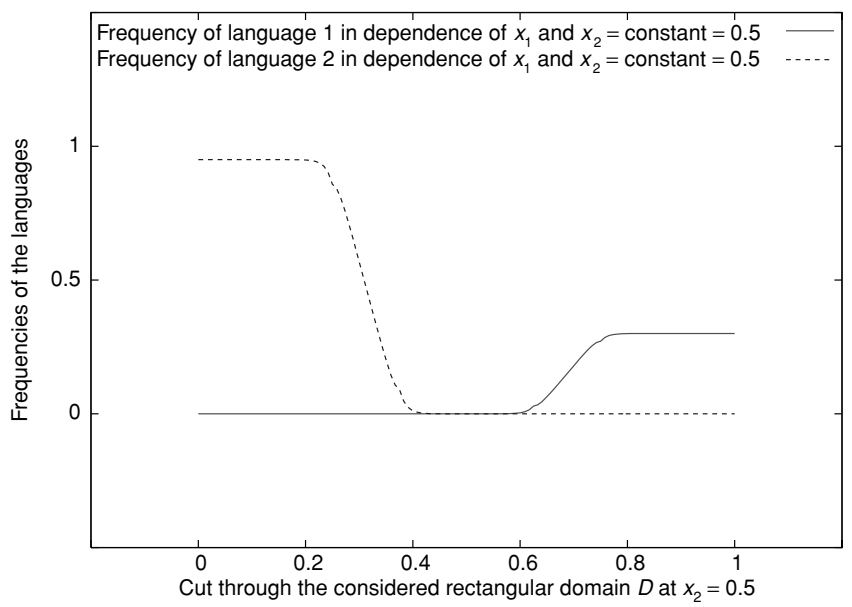

Figure 2.

Initial distribution of the frequencies.

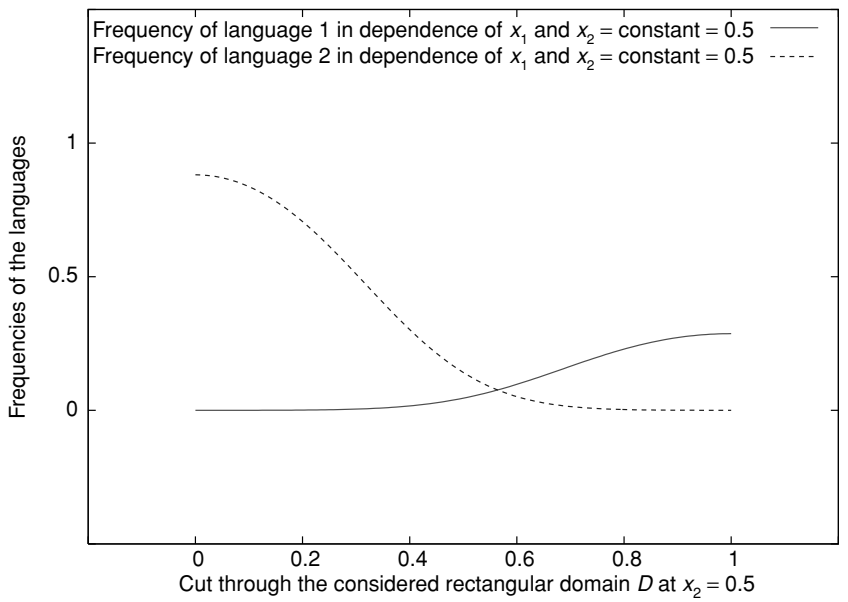

Figure 3.

Frequency distributions after 5 years.

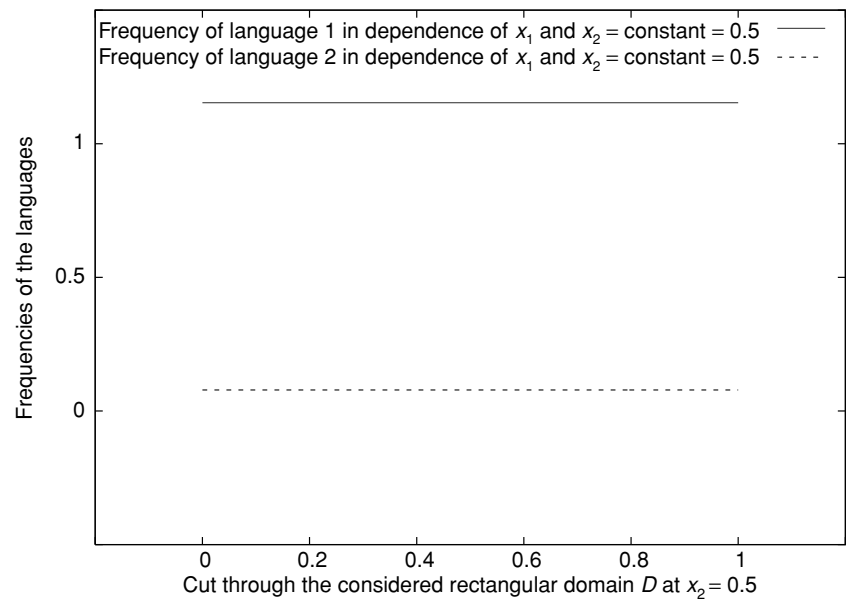

Figure 4.

Frequency distributions after 40 years. 


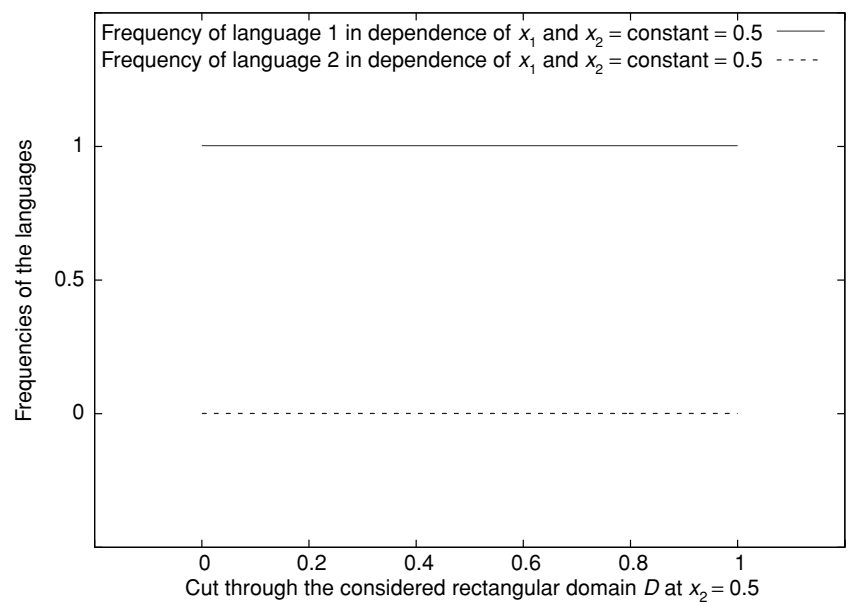

Figure 5.

Frequency distributions after 130 years.

carrying capacity $K_{1}$. This violation of the restriction persists until the frequency of language 2 is equal to zero (i.e., language 2 has become extinct), when the frequency $u_{1}$ returns to $K_{1}$. This effect violates the model assumption $K_{1}=u_{1}^{\max }$, and consequently the equilibrium $\left(K_{1}, 0\right)$ is not feasible.

Such a problem did not arise in the earlier models of Abrams and Strogatz (2003) and of Patriarca and Leppänen (2004), which required the total number of individuals (speaking whichever language) to be conserved. The reason for the aberrant behavior of the system in this two-species ecological model is the permanent conversion of speakers from language 2 to the dominant language 1 , in a system where (in the original two-species ecological context) the competition term is purely a loss term for the less competitive species. In the absence of competition, the populations of speakers of both languages would experience logistic growth, with the intrinsic growth parameter $a_{i}$ and the self-limiting coefficient $b_{i}=a_{i} / K_{i}$ maintaining $u_{i}$ at or below the carrying capacity $K_{i}$. But language 1 possesses the additional nonnegative "growth" term $c u_{1} u_{2}$ and the coefficient $b_{1}$ is now too weak to "save" the boundary $K_{1}$. As a consequence the frequency of language 1 exceeds its upper boundary value. Once language 2 becomes extinct then the competition term $c u_{1} u_{2}$ equals zero and the growth of language 1 is determined by the logistic growth function-the frequency $u_{1}$ tends toward the carrying capacity $K_{1}$.

Summarizing the results above we obtain that the equilibria $(0,0)$ and $\left(0, K_{2}\right)$ are not stable, and that the coexistence state

$$
\left(u_{1}^{*}, u_{2}^{*}\right)=\left(\frac{a_{2} K_{1}\left(a_{1}+c K_{2}\right)}{a_{1} a_{2}+c^{2} K_{1} K_{2}}, \frac{a_{1} K_{2}\left(a_{2}-c K_{1}\right)}{a_{1} a_{2}+c^{2} K_{1} K_{2}}\right)
$$

and the extinction state $\left(K_{1}, 0\right)$ are not feasible. This means that system (1) possesses no meaningful stable long-term ratios for the frequencies of both languages. To address this problem we now develop two modified versions of model (1).

\section{"Capped" Model}

In this section we modify the above model (1) in such a way that it is no longer possible for the number of speakers of language 1 to exceed their carrying capacity $K_{1}$. The idea is to adjust the self-limiting coefficient $b_{1}$ to take account of the dominant status of language 1 .

If we interpret $c u_{1} u_{2}$ as an additional growth term we can rewrite the term $u_{1}\left[a_{1}-b_{1} u_{1}+c u_{2}\right]$ in the form $u_{1}\left[a_{1}+c u_{2}\right]-\tilde{b}_{1} u_{1}$. With the following definition of the selflimiting coefficient $\tilde{b}_{1}=\tilde{b}_{1}\left(u_{2}\right)=\left(a_{1}+c u_{2}\right) / K_{1}$ the growth of the dominant language 1 is now restricted to the carrying capacity $K_{1}$. These considerations lead to the following slightly modified reaction-diffusion competition model

$$
\begin{aligned}
& \frac{\partial u_{1}}{\partial t}=d_{1} \Delta u_{1}+u_{1}\left[a_{1}-b_{1} u_{1}+c u_{2}\right]-\frac{c}{K_{1}} u_{1}^{2} u_{2}, \\
& \frac{\partial u_{2}}{\partial t}=d_{2} \Delta u_{2}+u_{2}\left[a_{2}-b_{2} u_{2}-c u_{1}\right] .
\end{aligned}
$$

The meaningful equilibria of this model in a bounded convex two-dimensional habitat $D$ have the form

$$
\begin{aligned}
& \left(u_{1}^{*}, u_{2}^{*}\right)=(0,0), \quad\left(u_{1}^{*}, u_{2}^{*}\right)=\left(K_{1}, 0\right), \\
& \left(u_{1}^{*}, u_{2}^{*}\right)=\left(0, K_{2}\right), \quad\left(u_{1}^{*}, u_{2}^{*}\right)=\left(K_{1}, \frac{a_{2} K_{2}-c K_{1} K_{2}}{a_{2}}\right) .
\end{aligned}
$$

Again the case of extinction of the total population of speakers of both languages $(0,0)$ is not of interest. The second and third equilibria indicate situations where one language becomes extinct. For the parameter constellation $a_{2}>c K_{1}$, which is derived from the assumption

$$
u_{2}^{*}=\frac{a_{2} K_{2}-c K_{1} K_{2}}{a_{2}} \geq 0,
$$

the fourth equilibrium describes a coexistence state with positive components $u_{1}^{*}$ and $u_{2}^{*}$. Language 1 is dominant and therefore its frequency grows until the carrying capacity $K_{1}$ is reached, but language 2 also has a positive frequency. The stability of these equilibria can be derived by applying phaseplane analysis. We obtain that-not surprisingly - the states $(0,0)$ and $\left(0, K_{2}\right)$ are unstable. The extinction state $\left(K_{1}, 0\right)$ is stable for the parameter constellation $a_{2}<c K_{1}$, which is the same condition as in model (1). In contrast, the coexistence state is now stable if $a_{2}>c K_{1}$ is fulfilled. This condition is equivalent to the "existence" condition (3) for the coexistence state. $^{3}$ This means that if this state exists in a meaningful sense then it is stable.

In analyzing the likelihood of loss of endangered languages it is also interesting to ask how long it takes until 

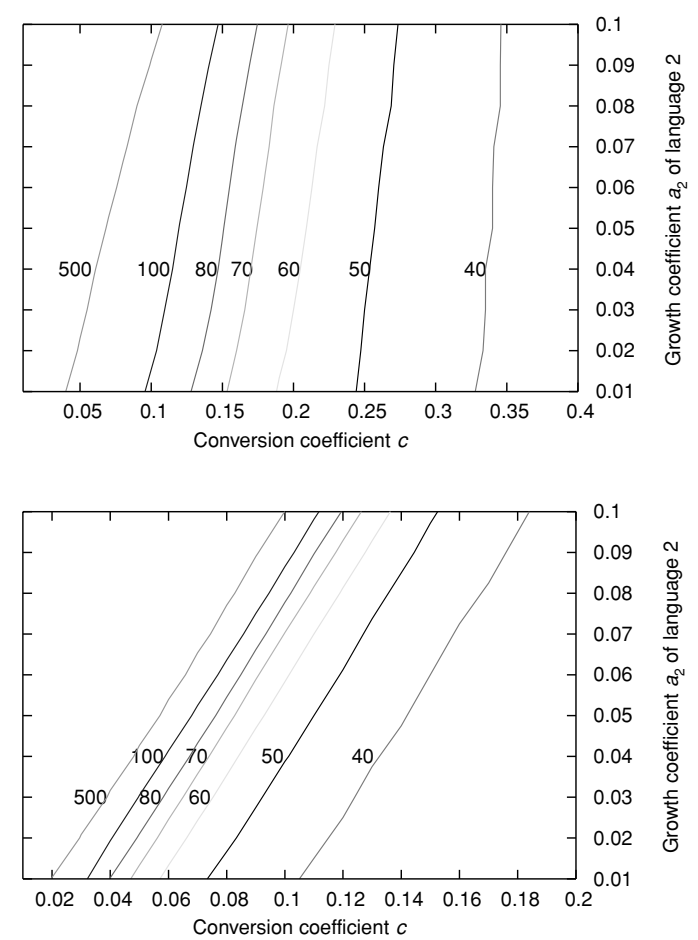

Figure 6.

Every line represents the parameter constellations of $a_{2}$ and $c$, which lead to the same extinction time (in years). In the left figure the growth coefficient of language 1 is chosen to be $a_{1}=0.01$ and in the right $a_{1}=0.25$. The diffusion coefficients are the same in both examples, $d_{1}=0.005$ and $d_{1}=0.001$.

the extinction states are reached. Figure 6 gives numerical estimates of extinction times if the growth rates of the higherstatus language 1 are assumed to be $a_{1}=0.01$ (Figure 6, left) and $a_{2}=0.25$ (Figure 6 , right). The carrying capacities $K_{1}$ and $K_{2}$ of both languages are chosen to be 1. In Figure 6 all curves are below the bisecting line that illustrates the extinction condition $a_{2}<c$. It is obvious that different parameter constellations $a_{2}$ and $c$ can lead to the same extinction time, but a higher growth coefficient $a_{2}$ needs to be balanced by a higher conversion rate $c$ to maintain the same extinction time. Additionally, if language 1 has a higher growth rate $a_{1}$ then smaller conversion rates $c$ are required to obtain the same extinction times.

In summary, this modified "capped" model (2) yields two stable equilibria in a bounded convex two-dimensional habitat $D$. Depending on the parameter constellation describing the language ecology, i.e., whether $a_{2}>c K_{1}$ or $a_{2}<c K_{1}$, we obtain either a coexistence situation or a situation where the endangered language 2 becomes extinct.

\section{Model With a Common Carrying Capacity}

In this section we discuss the use of the term "carrying capacity" in the previous models (1) and (2). These models are adopted from the field of population ecology. There the analysis of interactions between different species is the main con- cern. In this context and following Ehrlich (1994) the carrying capacities represent the maximum population sizes of species that an area can support without reducing its ability to support the same species in the future. But in the case of language competition we consider only one species carrying two different variants of one cultural trait. So unless socioeconomic factors create entirely separate niches for each language and its speakers, there should be only one upper boundary for population size - the maximum number of individuals which is supported by a given area, regardless of the language spoken.

In the following we assume that both languages compete for a finite pool of speakers, who have a common carrying capacity. That means that the frequencies of speakers of both languages have to fulfill the condition

$$
u_{1}+u_{2} \leq K
$$

where $K$ stands for the common carrying capacity. This condition changes the growth dynamics of both languages even in absence of competition. Assuming $u_{2}$ is fixed then the frequency of language 1 must be less than or equal to $K-u_{2}$, so that condition (4) is met. In other words, the frequencies $u_{1}$ and $u_{2}$ can grow only until (respectively) $K-u_{2}$ and $K-u_{1}$ are reached. This means that the self-limiting coefficient $\tilde{b}_{i}$ must be modeled so that it is able to restrict the frequency of speakers of language $i$ to $K-u_{j}$. With the definitions $\tilde{b}_{1}=\tilde{b}_{1}\left(u_{2}\right)=a_{1} /\left(K-u_{2}\right)$ and $\tilde{b}_{2}=\tilde{b}_{2}\left(u_{1}\right)=a_{2} /\left(K-u_{1}\right)$ we obtain the following model:

$$
\begin{aligned}
& \frac{\partial u_{1}}{\partial t}=d_{1} \Delta u_{1}+a_{1} u_{1}\left[1-u_{1} /\left(K-u_{2}\right)\right]+c u_{1} u_{2}, \\
& \frac{\partial u_{2}}{\partial t}=d_{2} \Delta u_{2}+a_{2} u_{2}\left[1-u_{2} /\left(K-u_{1}\right)\right]-c u_{1} u_{2} .
\end{aligned}
$$

In this model, violation of the boundary condition (4) due to the competition term is not possible since we have a symmetric situation. The loss of speakers of one language is the gain of the other language. The terms $\pm c u_{1} u_{2}$ do not change the total population frequency $u_{1}+u_{2}$.

The meaningful equilibria of model (5) in a bounded convex two-dimensional habitat $D$ are $\left(u_{1}^{*}, u_{2}^{*}\right)=$ $(0,0),\left(u_{1}^{*}, u_{2}^{*}\right)=(K, 0),\left(u_{1}^{*}, u_{2}^{*}\right)=(0, K)$ where only $(K, 0)$ is a stable equilibrium. The structure of model (5) implies that coexistence is no longer possible. Language 2 cannot resist the permanent conversion of speakers to language 1 since the situation described in note 3 cannot be reached. The condition $u_{1}+u_{2} \leq K$ implies that due to the permanent nonnegative influx of speakers language 1 will stop growing only if $u_{1}=K$ is reached. But this leads to the extinction of language 2. Figure 7 shows numerical estimates of the extinction times for different parameter constellations (i.e., for different environmental conditions). The growth rates of the dominant language are assumed to be $a_{1}=0.01$ (Figure 7, left) and $a_{2}=0.25$ (Figure 7, right), and the carrying capacity is set to be $K=1$. As 

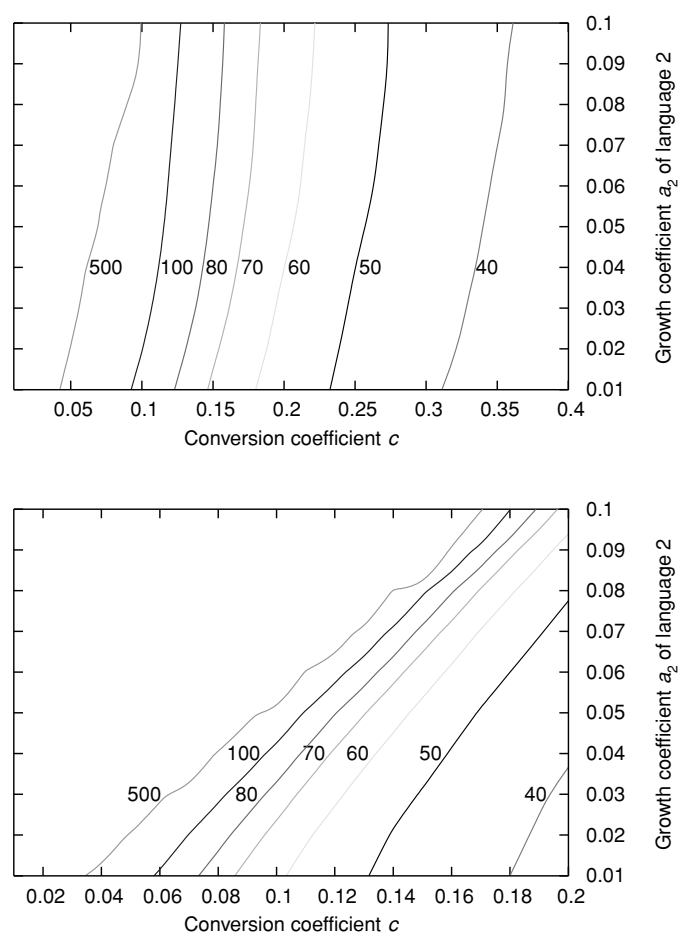

Figure 7.

Analogous to Figure 6 every line represents the parameter constellations of $a_{2}$ and $c$, which lead to the same extinction time (in years). In the left figure the growth coefficient of language 1 is chosen to be $a_{1}=0.01$ and in the right $a_{1}=0.25$. The diffusion coefficients are the same in both examples, $d_{1}=0.005$ and $d_{1}=0.001$.

in Figure 6 different values for $a_{2}$ and $c$ can lead to the same extinction times, and a higher growth coefficient $a_{2}$ must be balanced by a higher conversion rate $c$ to maintain the same extinction time. Furthermore, if language 1 has a higher growth rate then smaller conversion rates are again required to obtain the same extinction times.

Model (5) in a bounded convex two-dimensional habitat $D$ predicts the extinction of the lower-status language. The permanent loss of speakers through competition cannot be compensated by their internal reproductive rate. Even a large growth rate $a_{2}$ leads only to an extension of the time-course until the equilibrium $(K, 0)$ is reached. ${ }^{4}$

\section{Spatial Heterogeneity}

We have seen that under the assumption of a homogeneous environment model (5) leads to extinction of the lower-status language. In this section we want to explore the effects of spatial heterogeneity on the interaction dynamic and consider a situation where both competing languages are dominant in different core areas. The reasons for dominance in such core areas can relate to political, social, and/or economic factors. ${ }^{5}$ We incorporate this fact in model (5) by allowing the competition coefficient $c$ to vary spatially. We have shown in the previous section that every competitive advantage (modeled by an additive competition term) of one language leads to the extinction of the other over time. So we assume that the considered two-dimensional area $D$ is divided into subareas $D_{1}$ and $D_{2}$ where $^{6} c(x)=c_{1}$ if $x \in D_{1}$ and $c(x)=c_{2}$ if $x \in D_{2}$. The areas $D_{1}$ and $D_{2}$ are the core areas-the areas of dominance respectively of languages 1 and 2 .

In the following we consider the modified version of model (5) where $c(x)$ is chosen as defined above. At first we determine the dynamic of the modified model with $d_{1}=d_{2}=0$, which means the speakers of both languages are not allowed to move in space, with language 1 initially present in the whole area $D_{1}$ and language 2 in the whole area $D_{2}$. We already know that with the chosen competition coefficient $c(x)$, language 1 is dominant in area $D_{1}$ and language 2 in area $D_{2}$. Since both groups of speakers are assumed to be nondiffusive we obtain an equilibrium as shown in Figure 8. The spatial heterogeneity in the competition behavior of both languages results in an equilibrium which is no longer spatially constant and where both languages are spatially separated. Language 1 drives language 2 to extinction in the area $D_{1}$ and language 2 does the same with language 1 in area $D_{2}$.

We now consider the influences of spatial diffusion. The diffusion term characterizes the spatial dispersal of speakers of each language in our model and causes movement of speakers of language 1 into the area $D_{2}$ and vice versa. Due to the dominance of each language in its core area $\left(D_{1}\right.$ and $\left.D_{2}\right)$, each language is immune to extinction in its own core area. But the diffusion mechanism is able to produce a stable interaction or coexistence zone between the two core areas. Figures 911 show the stable long-term ratios of both languages for different values of the diffusion coefficients-a measure of the tendency to spread in space- $d_{1}$ and $d_{2}$. It is obvious that the equilibria are not spatially constant. The larger the diffusion coefficients, i.e., the further the speakers of one language are able to spread into the core area of the other language, the wider is the stable coexistence zone. Spatial spread can even mix both languages in such a way that they are both present over the whole considered area $D .^{7}$

Summarizing, the inclusion of spatial heterogeneity to model (5) in the form of spatial varying competition behavior leads to more complex situations, where-due to the assumed dominance of both languages in their core areas - coexistence is possible. The width of the interaction zone depends on the tendency of speakers of both languages to be spatially mobile outside their own core areas.

\section{Conclusion}

In this article we have considered different models of language competition in the case where one language is dominant. These situations are of particular interest since they can be found in many modern language-contact situations. We have seen that the ecological competition model shows some 


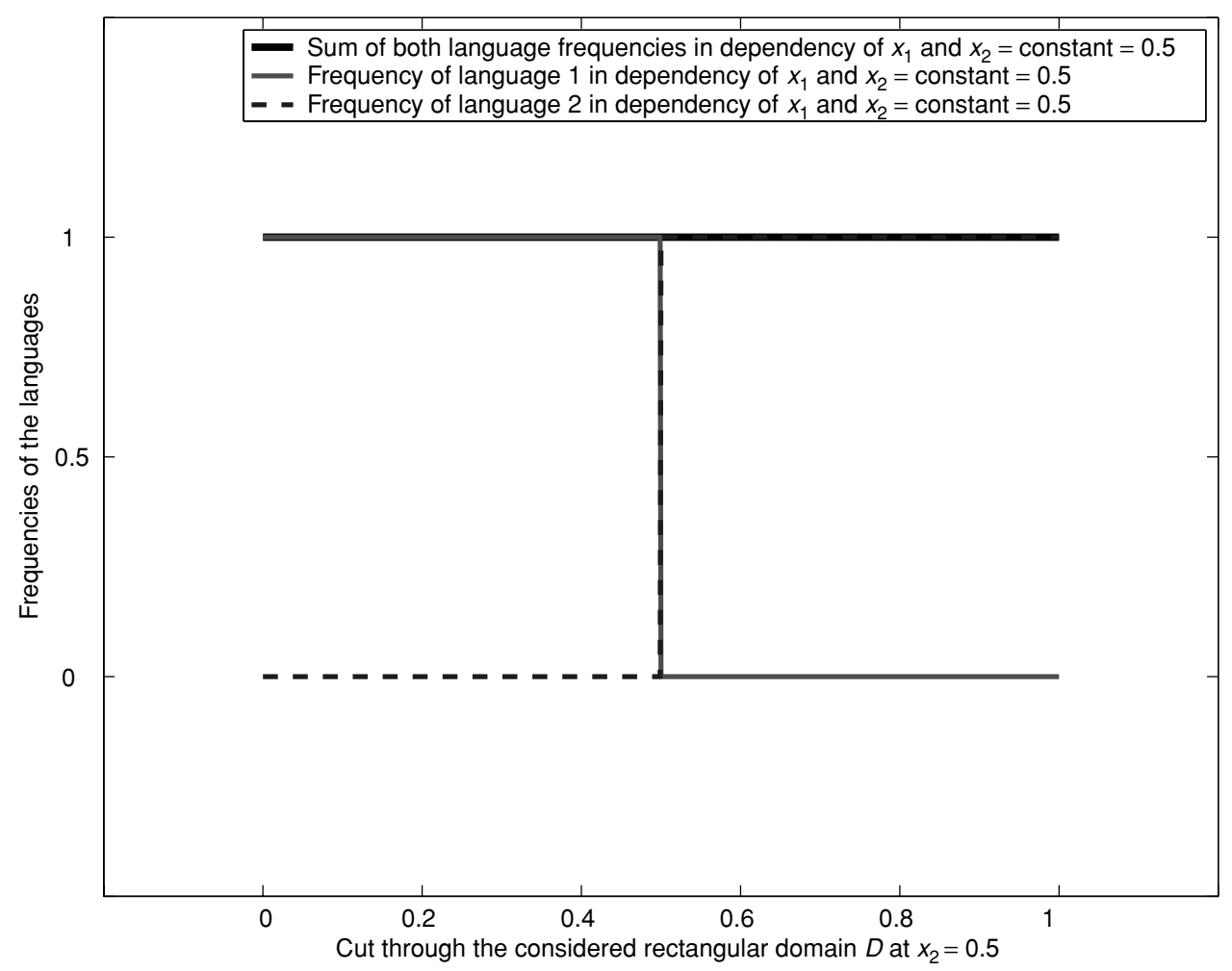

Figure 8.

Stable long-term ratios of both languages for $d_{1}=d_{2}=0$.

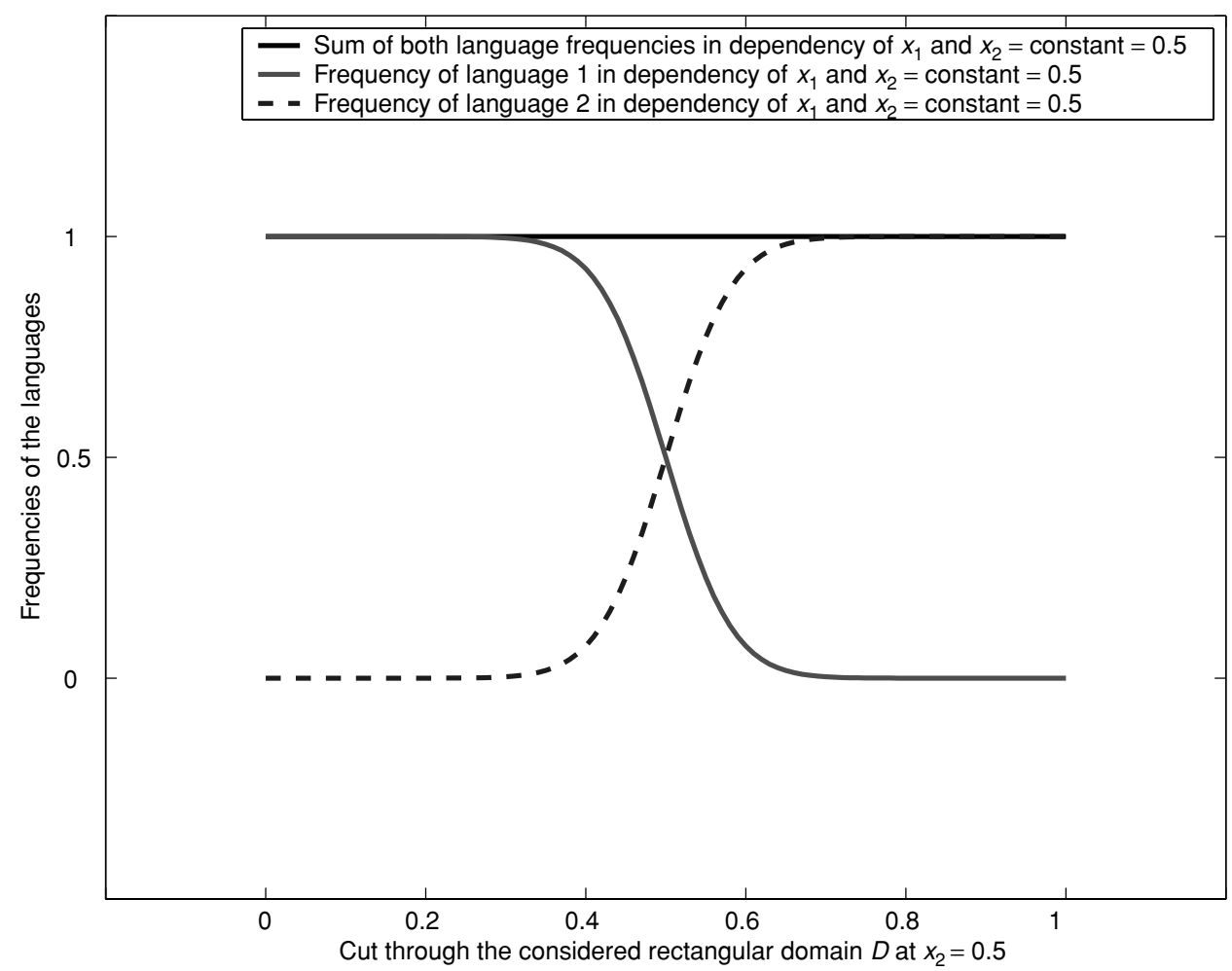

Figure 9.

Stable long-term ratios of both languages for $d_{1}=d_{2}=10^{-3}$. 


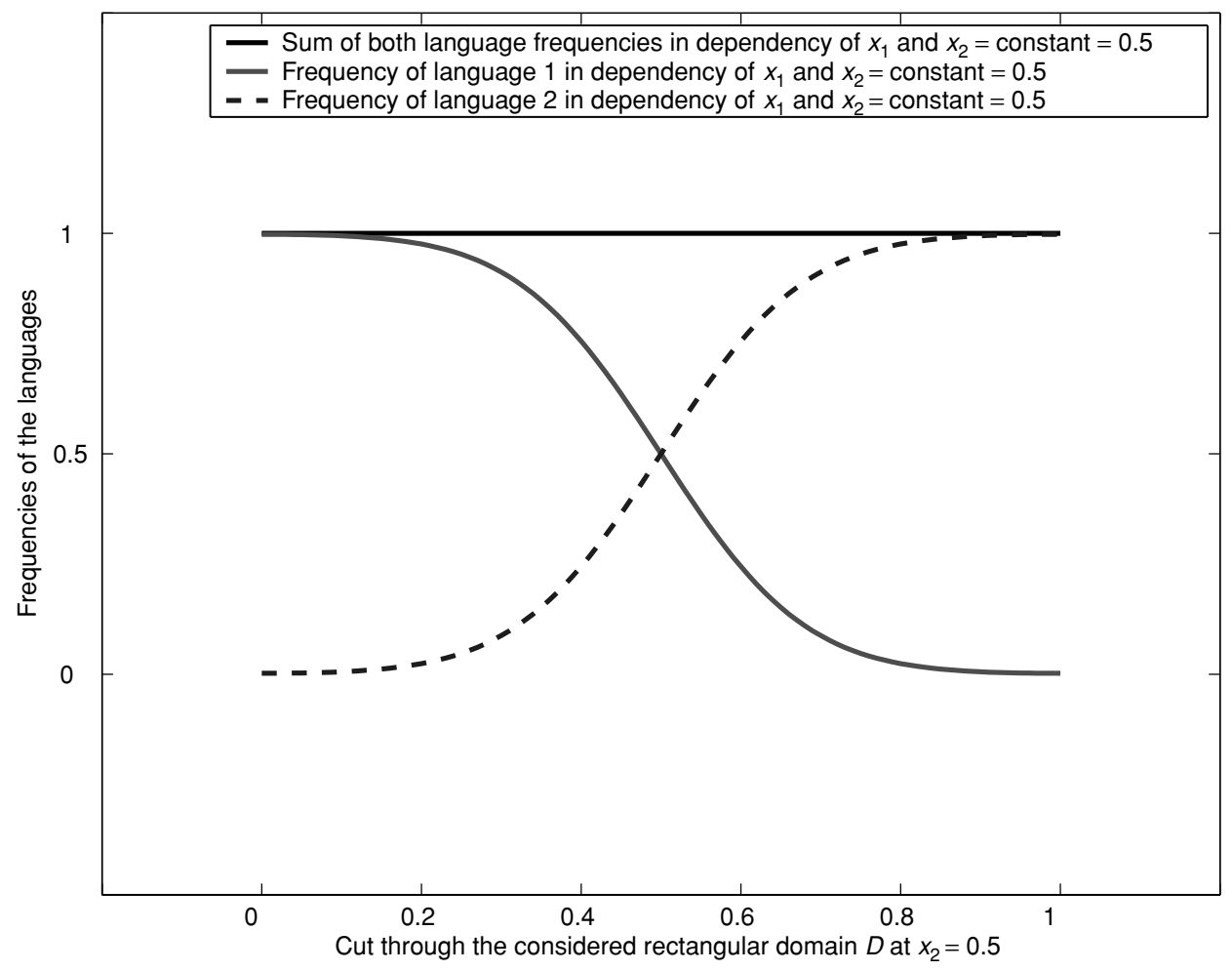

Figure 10.

Stable long-term ratios of both languages for $d_{1}=d_{2}=10^{-2}$.

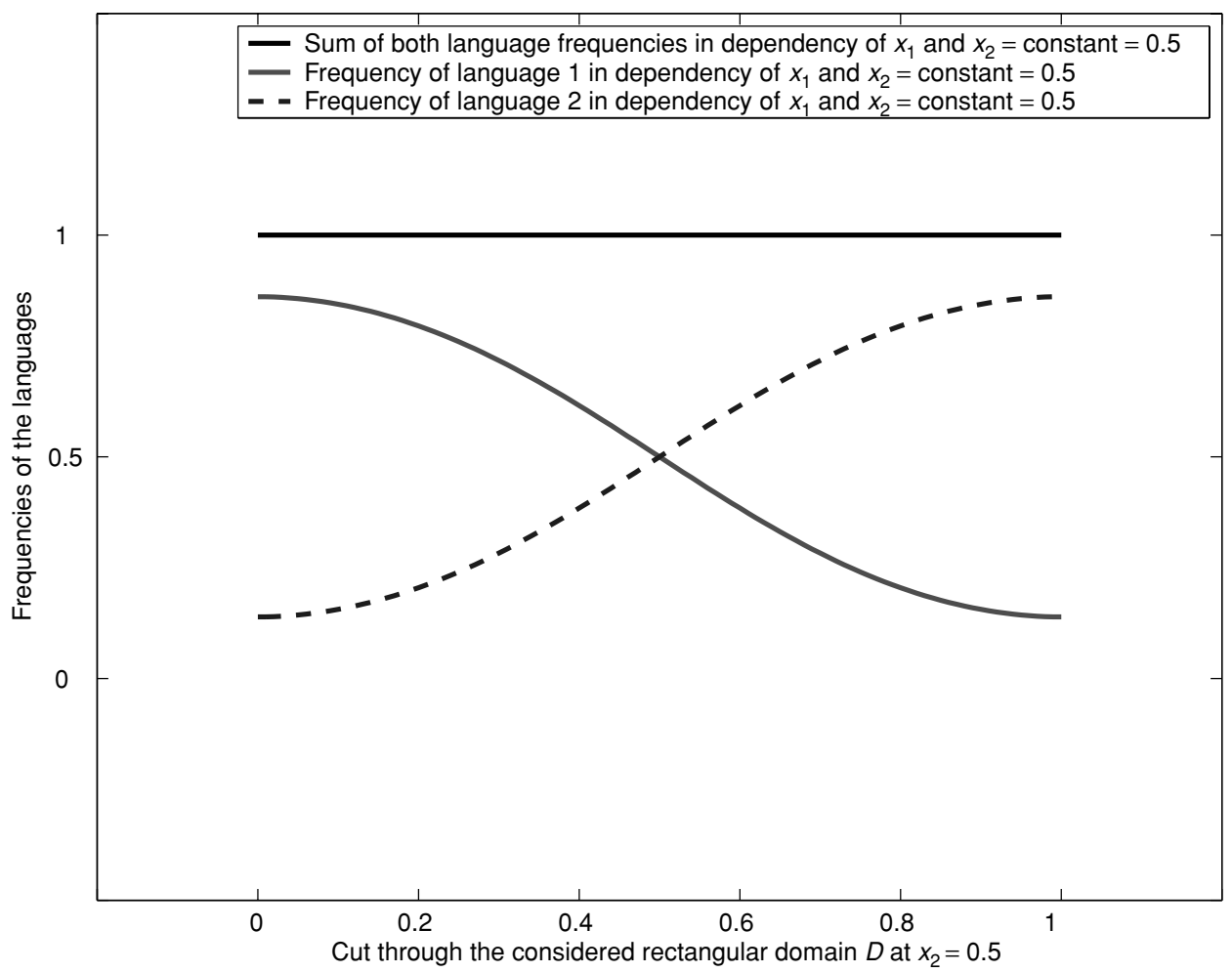

Figure 11.

Stable long-term ratios of both languages for $d_{1}=d_{2}=10^{-1}$. 
deficiencies when applied to language competition. We have modified this model to preserve the boundary condition for maximum numbers of speakers of the dominant language. This modified model predicts that different environmental conditions can support states of coexistence as well as states of extinction of the endangered language. However, this modified model still assumes that competing languages have separate carrying capacities for their speakers. This seems an unrealistic assumption since languages can be considered as different variants of the cultural trait, "language." We therefore developed a third model in which both languages compete for a common population of potential speakers. In this model, as in the original model of Abrams and Strogatz (2003), the extinction of an endangered language is predicted where there is preferential shifting into the dominant language by its speakers. But adding spatial heterogeneity in the competition term leads to more complex situations where coexistence of languages is possible.

\section{Notes}

1. A number of other mathematical approaches to language competition exist, including agent-based models (Castelló et al. 2007) and Monte Carlo simulations based on game theory (Kosmidis et al. 2005), some of which also consider bilingualism (Baggs and Freedman 1993; Castelló et al. 2007). Schulze and Stauffer (2006)is a recent review of such work by physicists.

2. We note that the reaction-diffusion system (1) in a bounded convex domain leads to the same equilibria as the ODE system, without considering the diffusion component (which yields only transient effects). However, in the real-world context, language competition is usually an outcome of new or intensified contact between populations that retain some initial spatial separation. Our method of solution permits us to observe the effects of different levels of diffusivity on the time course to these equilibria, and would also allow us to modify the spatial structure of the domain in order to study the effects of further confining the zone of interaction.

3. The "existence" and stability condition $a_{2}>c K_{1}$ for the coexistence state can be interpreted as follows: The term $c u_{1} u_{2}$ describes the conversion rate of speakers from language 2 to language 1 . So the conversion rate is proportional to the product of the actual frequencies of the languages. Further $K_{1}$ is the upper boundary for the frequency of speakers of language 1 . In the case $u_{1}=K_{1}$ language 1 exerts the highest pressure on language 2 . But if speakers of language 2 can compensate for these peak loss rates by a sufficiently high reproductive rate, then language 2 can still survive. And this is exactly what $a_{2}>c K_{1}$ implies.

4. This result could be inverted by assuming density-dependent conversion parameters, which would — contrary to the considerations so far-imply that the advantage of a language is determined by its frequency. If, for example, a rapid dispersal of its speakers caused the initially high-status language to fall below a certain population density threshold in a specific area, then the sign of the conversion term would change in that area and the former low-status language becomes the high-status language (and vice versa).
5. An example is the situation in the Basque Country, which enjoys substantial cultural and political autonomy, and where the Basque language is protected by laws - and by the regulatory activity of an academy set up to maintain it. The Basque language is an isolate, unrelated to the Indo-European languages also spoken in this and adjacent regions. It is plausible that this makes it more difficult for speakers in one group to learn the other group's language, and that this has also impeded rates of shifting (although we do not explicitly consider such factors here).

6. For analytical reasons $c(x)$ is not implemented as a jump function, we model $c(x)$ as a linear function in a narrow transition zone. It is not oversimplifying to treat the function $c(x)$ as constant within the different core areas because which language is locally dominant depends only on the sign of $c$ and not on the numerical value.

7. Situations with different values for the diffusion coefficients $d_{1}$ and $d_{2}$ result in asymmetric coexistence zones.

\section{References}

Abrams DM, Strogatz SH (2003) Modelling the dynamic of language death. Nature 424: 900.

Baggs I, Freedman HI (1993) Can the speakers of a dominated language survive as unilinguals?: A mathematical model of bilingualism. Mathematical Computer Modelling 18(6): 9-18.

Castelló X, Loureiro-Porto L, Eguíluz V, San Miguel M (2007) The fate of bilingualism in a model of language competition. In: Advancing Social Simulation: The First World Congress (Takahaski S, Sallach D, Rouchier J, eds), 83-94. New York: Springer.

Cosner C, Lazer AC (1984) Stable coexistence states in the Volterra-Lotka competition model with diffusion. SIAM Journal on Applied Mathematics 44: 1112-1132.

Ehrlich PR (1994) Ecological economics and the carrying capacity of the Earth. In: Investing in Natural Capital: The Ecological Economics Approach to Sustainability (Jansson AM, ed), 38-56. Washington, DC: Island Press.

Kosmidis K, Halley JM, Argyrakis P (2005) Language evolution and population dynamics in a system of two interacting species. Physica A 353: 595-612.

Krauss M (1992) The world's languages in crisis. Language 68: 4-10.

Mufwene S (2002) Colonisation, globalisation, and the future of languages in the twenty-first century. International Journal on Multicultural Societies 4: 162-193.

Muhlhausler P (2000) Language planning and language ecology. Current Issues in Language Planning 1: 306-367.

Murray JD (2002) Mathematical Biology I, 3rd ed. New York: Springer.

Patriarca M, Leppänen T (2004) Modeling language competition. Physica A 338: 296-299.

Pinasco JP, Romanelli L (2006) Coexistence of language is possible. Physica A $361: 355-360$.

Schulze C, Stauffer D (2006) Recent developments in computer simulations of language competition. Computing in Science and Engineering 8(3): $60-67$. 